\title{
Dieta, actividad física y estado de nutrición en escolares tarahumaras, México
}

MARTHA BALCÁZA ${ }^{(1)}$, PATRICK PASQUET ${ }^{(2)}$ e IGOR DE GARINE ${ }^{(2)}$

\section{RESUMEN}

Objetivo: El objetivo del presente estudio fue describir y comparar la dieta, la actividad física y el estado de nutrición en niños indigenas tarahumaras de edad escolar de tres grupos: Tarahumaras tradicionales, tarahumaras semi-tradicionales y tarahumaras urbanos migrantes originarios del mismo municipio. Material y método: Se realizó un estudio prospectivo, descriptivo, analítico y comparativo, de corte transversal, aplicado a 193 niños en tres grupos de ambos sexos de 6 a 12 años. Fueron aplicados recordatorios de 24 horas, pesos y actividad física con acelerometría, antropometría nutricional y mediciones bioquímicas. Resultados: Se encontró retraso en crecimiento principalmente en el grupo tradicional. Existe una marcada anemia en el grupo migrante. Han sido observados casos de sobrepeso en niños semi-tradicionales. Se encontró un elevado porcentaje de sobrepeso y obesidad en niños urbanos. Estas diferencias se explican más particularmente por variaciones en el patrón de consumo alimentario. Conclusión: se puede concluir que se observan cambios nutricionales en niños tarahumaras, que son más evidentes en zonas rurales, similares a muchas sociedades en transición económica. Este proceso de "desindigenización" o "mestizaje" de los patrones dietéticos tiene que ser analizado antes de implementar nuevas politicas a esta población indigena.

Palabras clave: Transición nutricional, actividad física, obesidad.

\section{ABSTRACT}

\section{DIET, PHYSICAL ACTIVITY AND NUTRITION STATE IN TARAHUMARA SCHOOLCHILDREN IN MEXICO}

Objective: The objective of this study was to describe and compare the diet, physical activity and nutrition state in school age, indigenous Tarahumaras from three groups: traditional Tarahumara, semi-traditional Tarahumara and urban migrants originally from the same municipality. Materials and methods: This was a cross sectional, prospective, descriptive, analytic and comparative study, using 193 children in the three groups, both sexes, from 6 to 12 years old. We used 24 hours reminders, weights, physical activity with accelerometers, nutritional anthropometry and biochemical measurements. Results: We found growth delay mainly in the traditional group. There was marked anemia in the migrant group. We observed cases of overweight in children from the semi-traditional group, and an elevated percentage of obesity and overweight was found in the urban migrant group. These differences are explained in particular by variations in eating patterns. Conclusion: Nutritional changes were observed in Tarahumara children, and are more evident in rural zones, similar to many other societies in economic transition. This process of "deindigenization" or

(1) Departamento de Salud. Universidad Iberoamericana. Prol. Paseo de la Reforma 880. Lomas de Santa Fe. DF. CP 01219. Cd. México. México. martha.balcazar@uia.mx

(2) UMR 5145. Centre National de la Recherche Scientifique. Paris. Francia. 
Dieta, actividad física y estado de nutrición en escolares tarahumaras, México - Martha Balcáza et al

"mixing" of dietary patterns must be analyzed before implementing new policies targeted at this indigenous population.

Keywords: nutritional transition, physical activity, obesity.

\section{INTRODUCCIÓN}

El grupo indígena tarahumara o "rarámuri" como ellos se denominan, vive en una situación de pobre salud, aislamiento y exclusión social. Esta población indígena es la más numerosa en el norte de México y una de las minorías étnicas con mayor marginación en el país.

Sus pobladores cuentan con una gran resistencia a condiciones ambientales extremas y a demandas de ejercicio de máximo rendimiento, afectando esto su estado de nutrición. Esta población vive en la sierra del estado de Chihuahua, donde escasea el agua y las condiciones climáticas son extremosas. Viven en asentamientos dispersos en montañas muy elevadas y frías, donde también hay prolongadas sequías.

Una expresión vigente de esta resistencia es la "carrera de bola" tradicional, o "rarajipari" en la cual los hombres recorren distancias que llegan a sobrepasar los $150 \mathrm{~km}$. atravesando montañas y valles.

Esta cualidad de intensidad en su actividad física se inicia con los niños, cuyo trabajo cotidiano consiste en acarrear cabras o chivas para pastar. Esta actividad física dura de 6 a 8 horas en recorridos estimados de 4 a $25 \mathrm{~km}$. diarios.

Existe poca información de salud y nutrición de la población indígena en general. Dentro de ésta, se observa que los niños indígenas en el país se caracterizan por tener retraso del crecimiento, anemia, así como deficiencia de micronutrimentos, explicados por sus condiciones socioeconómicas ${ }^{1}$. A los tarahumaras se les considera una población que presenta desnutrición crónica ${ }^{2,3}$. Un trabajo en albergues escolares públicos con escolares taharumaras, en el municipio de Guachochi, indicó que hay desnutrición y deficiencia de micronutrimentos como el hierro, zinc, vitamina B12 y yodo, pero con una prevalencia menor a la esperada para este grupo de población indígena ${ }^{4}$.

Actualmente, como sucede en poblaciones de economía transicional, a estas deficiencias se suman problemas de sobrepeso y obesidad. Por ejemplo, en un estudio se evaluó a mujeres de albergues tarahumaras, en edad reproductiva, encontrando que $52.2 \%$ tiene sobrepeso ${ }^{5}$. Además, los tarahumaras son susceptibles al cambio de patrón de su dieta tradicional, y dichos cambios pueden incrementar su riesgo a desarrollar enfermedades crónico degenerativas ${ }^{6}$.

En una investigación reciente, se encontró una baja prevalencia de desnutrición y algunos casos de sobrepeso en niños tarahumaras, queprobablemente- refleja cambios en los hábitos alimentarios o una transición en la dieta ${ }^{7}$.

No hay duda de que los cambios en la alimentación global se están dando de una manera acelerada. Los casos de transición alimentaria de comunidades indígenas en Brasil y China son una clara muestra de ello ${ }^{8}$. Estos cambios ocurren principalmente porque hay un incremento en la densidad energética de su dieta y una ingesta reducida de muchos nutrimentos importantes, como aquellos que contienen fibra. La diversidad de alimentos se ha incrementado, y la desnutrición está cediendo en varias comunidades, sin embargo los hallazgos en poblaciones de tipo indígena son incipientes. Adicionalmente, la migración nacional e internacional de estas comunidades ha afectado profundamente la tendencia de la transición alimentaria.

Es por estas razones que se trabajó en esta población en su medio tradicional, semitradicional y con los migrantes a grandes centros urbanos, con el objeto de describir y comparar la dieta, la actividad física y el estado de nutrición en niños tarahumaras en edad escolar, así como definir los criterios de alimentación pertinentes, culturalmente aceptables, para establecer recomendaciones que disminuyan los riesgos alimenticios vinculados a la transición de modo de vida y comportamiento alimentario en los niños. 


\section{MATERIAL Y MÉTODO}

La población estudiada corresponde a una muestra de 193 escolares de ambos sexos de 6 a 12 años dividiendo a las muestras de los siguientes grupos poblacionales en:

1. Niños tarahumaras tradicionales, constituido por escolares que viven en la sierra, no reciben educación formal ni programas de ayuda alimentaria, habitan en las rancherías del pueblo de Norogachi, llamadas Pawichike y Neoachi. La mayoría es pastor de cabras y acarrea leña a su hogar.

2. Niños tarahumaras semi-tradicionales, integrado por escolares que asisten a un internado privado en Norogachi, localizado a 2,140 m de altitud, en el Municipio de Guachochi (Estado de Chihuahua, Norte de México), que es una comunidad central con servicios de salud, educación, religión y de comercio.

3. Niños tarahumaras urbanos, conformado por niños cuyas familias han migrado del mismo municipio en la sierra (Guachochi), a la ciudad de Chihuahua.

Los escolares seleccionados aleatoriamente fueron admitidos en el grupo de trabajo después de una valoración médica.

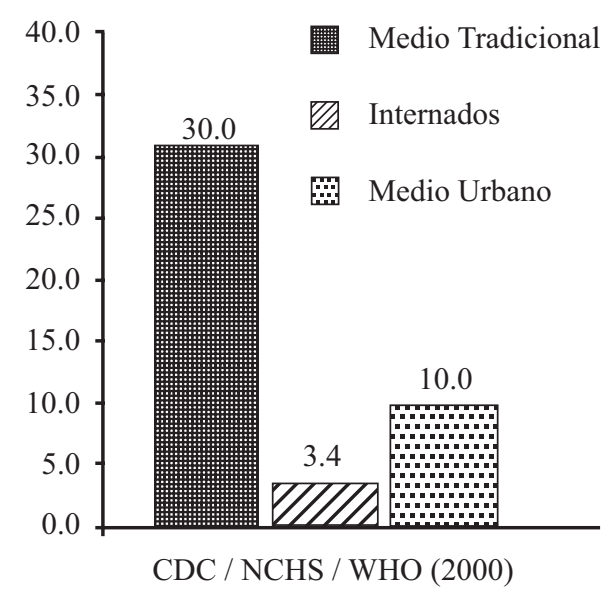

Figura 1. Retraso del crecimiento de niños tarahumaras $(\%)$.

\section{Análisis de dieta}

A los niños semi-tradicionales se les aplicó una encuesta de dieta de pesos y medidas por tres días en el internado. Los alimentos fueron pesados por asistentes especialmente capacitados, trabajando las 24 horas durante los periodos de medición. (Bascula digital SECA 852, Hamburgo, Alemania), pesando $10 \mathrm{~kg}$ con una precisión de $5 \mathrm{~g}$. Los alimentos eran pesados durante su preparación e ingeridos. La comida de recreo, dulces, fruta o refrigerios, fue también pesada antes de la ingestión. El vómito fue tomado en cuenta. A los niños tradicionales y urbanos se les aplicaron encuestas recordatorio de 24 horas por 3 días Ambas metodologías se siguieron tomando en cuenta el procedimiento estandarizado, diseñado y validado por el Instituto $\mathrm{Na}$ cional de Ciencias Médicas y Nutrición Salvador Zubirá9.

\section{Determinación antropométrica}

Se tomaron una serie de medidas antropométricas, por personal capacitado que se estandarizó mediante procedimientos convencionales $^{10,11}$. La talla se midió con un estadímetro portátil (Siber Hegner, Suiza). El peso de cada participante se tomó con ropa ligera, usando una báscula digital (Tanita, Japón). El Índice de Masa Corporal (IMC) fue calculado dividiendo el peso en $\mathrm{Kg}$ por el cuadrado de la talla en metros.

El estado nutricional fue determinado mediante índices antropométricos con mediciones de peso, talla y edad ${ }^{12}$. Los tres indicadores empleados fueron el peso esperado para la edad, la talla esperada para la edad y el peso esperado para la talla, y distribuciones de IMC usando la referencia OMS-NCHS-CDC ${ }^{13,14}$.

\section{Hemoglobina sanguínea}

La concentración de hemoglobina fue obtenida con una medición hemométrica, usando un analizador portátil Hemocue (EQT Partners $\mathrm{AB}$, Suecia), a partir de una gota de sangre capilar obtenida de punciones digitales. La anemia fue determinada considerando los puntos de corte recomendados por la OMS: $<11.5 \mathrm{~g} / \mathrm{dl}$ para niños de 7-11 años $\mathrm{y}<12.0 \mathrm{~g} / \mathrm{dl}$ para niños 
de 12 años ${ }^{15}$. Estos valores fueron ajustados por altitud sobre el nivel del mar considerando un incremento de $0.8 \mathrm{~g} / \mathrm{dl}$ de acuerdo a fórmulas de Cohen y Hass ${ }^{16}$.

\section{Determinación de actividad física diaria}

La actividad física diaria fue determinada durante lapsos de dos a tres días de escuela así como los dos días de fin de semana con acelerómetros unidireccionales CSA Actigraph AM7164 (MIT, FL), que han sido ampliamente validados en niños ${ }^{17}$. Los acelerómetros fueron fijados en la cadera izquierda y fueron usados continuamente por los niños, excepto para bañarse. Las cuentas de aceleración fueron acumuladas por una frecuencia de un minuto y el total se almacenó en la memoria. Se registraron las cuentas totales diarias (de 24 horas). Los puntos de corte fueron la determinación de 4 niveles de actividad: mínimo ( $<50$ cuentas), sedentario ( $\geq 50$ y $<800$ cuentas), ligero $(\geq 800$ $\mathrm{y}<3200$ cuentas) y moderado a vigoroso $(\geq 3200$ cuentas).

\section{Análisis estadístico}

Todos los datos analizados y el cálculo hecho fue con el software ESTATISTICA versión 6 (Statsoft, Inc, Tulsa, OK, USA). Los resultados descriptivos son presentados como media (DE).

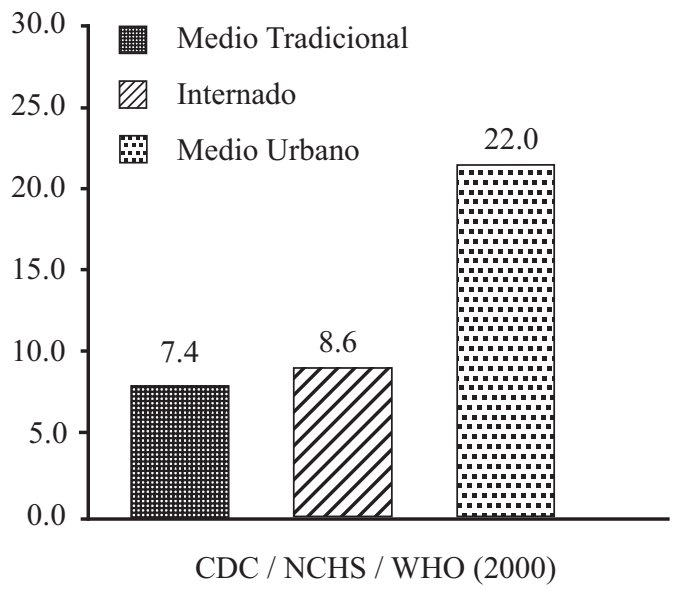

Figura 2. Sobrepeso y obesidad en niños tarahumaras $(\%)$
En la comparación de los grupos se usó la prueba $\mathrm{T}$ student, excepto las variables de la composición corporal, en donde se usó la prueba no paramétrica Mann-Whitney.

\section{Ética}

Todas las mediciones en este estudio se realizaron de acuerdo al Código de Ética de la Asociación Mundial Médica (Declaración de Helsinki). Un formato de consentimiento fue firmado por los niños y sus padres, después de haber sido totalmente informados de las metas y procedimientos del estudio. Este proyecto fue aceptado por la Comisión de Investigación y Comité de Ética del Instituto Nacional de Perinatología Isidro Espinosa de los Reyes, dependiente de la Secretaría de Salud, México.

\section{RESULTADOS}

Se trata de una población de 190 niños, a los cuales se les realizó un examen médico para ser tomado como parte de la muestra. De ellos, 187 fueron considerados clínicamente sanos y la muestra final del estudio consistió en 67 niños tradicionales, 58 semi-tradicionales y 62 urbanos. (Tabla 1).

Existe un retraso del crecimiento del 30\% en el grupo de niños tradicionales. (Figura 1). El sobrepeso entre los niños tradicionales y semi-tradicionales es bajo, de 7.4 y $8.6 \%$ res-

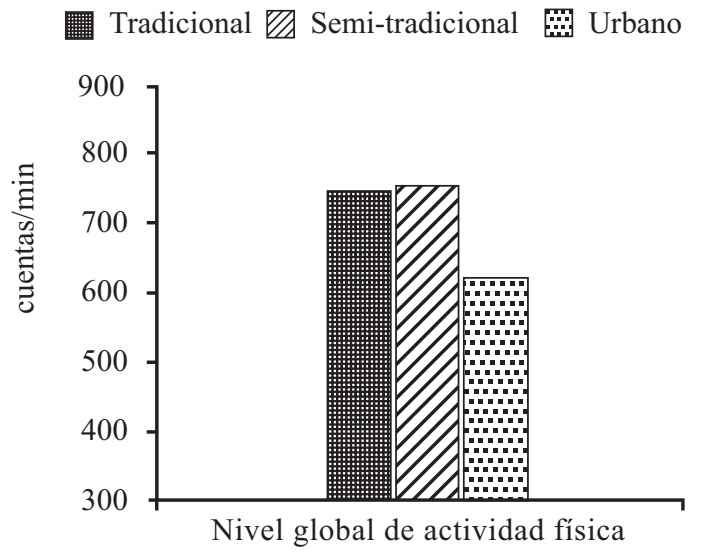

Figura 3. Nivel Global de Actividad Física en las tres muestras de población tarahumara. 
pectivamente. En los niños urbanos fue de 22\% (Figura 2).

En cuanto al consumo energético, los niños tradicionales presentan un consumo de calorías por debajo del requerimiento nutrimental. (Tabla 2). El mayor aporte de energía para los niños tradicionales es a partir de hidratos de carbono y sólo el $16 \%$ es proporcionado por lípidos.

Los niños tradicionales y urbanos presentan menor consumo de micro-nutrimentos de acuerdo a los requerimientos promedio establecidos para ácido fólico y vitamina $\mathrm{A}$, teniendo estos últimos el menor consumo también de calcio y hierro. Existe una baja ingesta de hierro en el grupo urbano que corresponde también a la medición de hemoglobina en los niños, de los cuales 33\% manifestó anemia; en el medio tradicional este padecimiento se presentó en el 10\% de los niños y $6 \%$ en niños semi-tradicionales.

Igualmente, los niños urbanos muestran el menor consumo de fibra, seguidos por los semitradicionales, hasta llegar a los tradicionales, que consumen más de cuatro veces fibra que los urbanos, los cuales sólo consumen una tercera parte de fibra comparados con los del grupo semi-tradicional.

Según la Figura 3, los niños urbanos tienen menor nivel de actividad física respecto a los otros grupos, siendo el grupo mas activo el de los niños semi-tradicionales.

La Figura 4 muestra los cuatro niveles de actividad física en los tres grupos, donde la actividad mínima de los tradicionales, semitradicionales y urbanos es similar debido que todos duermen aproximadamente ocho horas y media.

La actividad sedentaria es más alta en el grupo urbano, en la sierra predomina la actividad ligera, mientras que en los niños semitradicionales, es más notoria la actividad moderada a intensa aunque no es tan grande la diferencia con respecto a los otros 2 grupos.

En una cuantificación del porcentaje de niños con un mínimo de 60 minutos diarios en actividades moderadas a intensas, se encontró que los niños semi-tradicionales muestran los valores más altos en este rubro (Figura 5).

\section{DISCUSIÓN}

El retraso del crecimiento en los niños tradicionales de $30.0 \%$ fue superior al valor para la población mexicana, que fue de $10.4 \%$. También es superior al valor de la región sur del país con alto desmedro o prevalencia de baja talla. El retraso del crecimiento en el grupo del internado fue de $3.4 \%$, debido a la ayuda alimentaria, educación y servicios de salud que se dan en la

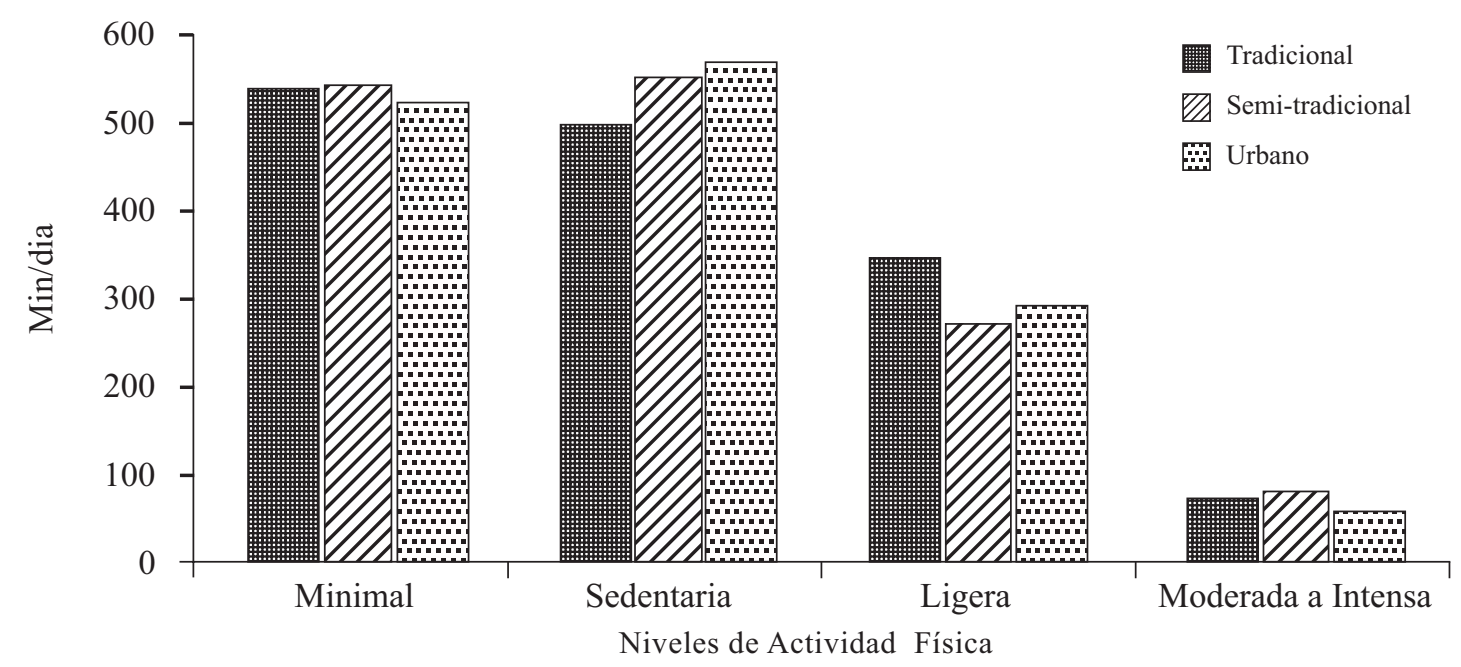

Figura 4. Niveles Específicos de Actividad Física en las tres muestras de población tarahumara. 


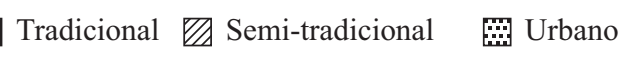

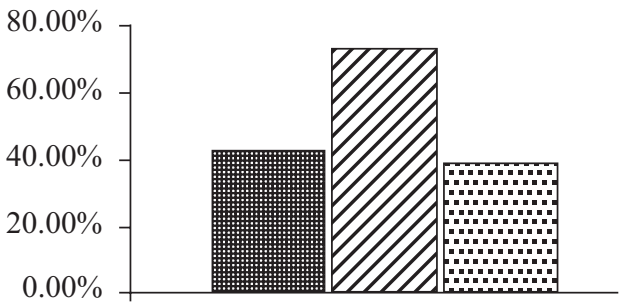

Figura 5. Porcentaje de niños con un mínimo de 60 minutos diarios en actividades moderadas a intensas.

comunidad de Norogachi. En cuanto al estrato urbano, uno de cada diez niños tiene un retraso en su crecimiento, el sobrepeso más la obesidad fue de $22 \%$, inferior a la cifra nacional, de $26 \%{ }^{17}$.

Los niños tradicionales exhiben una alta proporción de hidratos de carbono en su aporte energético, debido al autoconsumo de maíz, que es de los principales elementos de su dieta. En los niños semi-tradicionales, la composición de la dieta y el aporte de macro-nutrimentos, es cercano a la dieta recomendada y, por lo tanto, es equilibrada. Sin embargo, en los niños urbanos el consumo de macro-nutrimentos se acerca a los requerimientos con excepción de los lípidos que están por encima de lo recomendado señalando una tendencia al aumento del consumo de grasa a partir de la dieta.

El mayor consumo de fibra es por parte de los niños tradicionales y el menor es por parte de los niños urbanos, lo que repercute en la disminución de la bio-disponibilidad de nutrimentos.

Los niños semi-tradicionales presentan los mayores consumos de micro-nutrimentos por arriba de los requerimientos, especialmente ácido fólico, hierro, vitamina A y zinc, siendo esto consistente con el consumo diario de cereales, galletas y dulces adicionado de estos nutrimentos. Los niños urbanos exhiben menores consumos de micro-nutrimentos debido a su alto consumo de alimentos industrializados. Esto, a su vez, explica una mayor incidencia de anemia en este grupo.

La alimentación de los niños tradicionales, basada en frijol, maíz y quelites, les aporta una cantidad importante de fibra. En contraste, los niños urbanos exhiben un consumo mucho menor, principalmente por el escaso consumo de frutas y alimentos naturales altos en fibra.

La actividad física moderada a intensa es más baja en los niños tradicionales debido a que llevan un ritmo más lento de vida, para los niños urbanos son más bajos debido al sedentarismo característico de la urbe. En el caso de los niños semi-tradicionales, la actividad física es la mayor de entre los grupos, pues su educación incluye tiempos amplios de ejercicio físico.

Se encontró que los niños tradicionales mantienen el patrón tres de Popkin de transición alimentaria porque es una dieta sin variedad, monótona, baja en grasa, alta en fibra, y la

Tabla 1. Características de los escolares tarahumaras.*

\begin{tabular}{|l|c|c|c|}
\hline & Tradicionales & Semi-tradicionales & Urbanos \\
\hline & $\mathrm{n}=67$ & $\mathrm{n}=58$ & $\mathrm{n}=62$ \\
\hline Edad, años & $9.0(2.2)$ & $9.7(1.8)$ & $9.4(2.0)$ \\
\hline$\%$ de Mujeres & 43.3 & 50.8 & 52.9 \\
\hline$\%$ de Hombres & 56.7 & 49.2 & 47.1 \\
\hline Estatura, cm & $124.8(12.8)$ & $132.0(9.9)$ & $128.1(13.6)$ \\
\hline Peso Corporal, kg & $25.9(6.9)$ & $30.0(7.2)$ & $30.0(9.2)$ \\
\hline Indice de Masa Corporal $\left(\mathrm{IMC}, \mathrm{kg} / \mathrm{m}^{2}\right)$ & $16.33(1.36)$ & $16.98(1.77)$ & $17.55(2.35)$ \\
\hline Talla para la edad, \% & $19.8(22.5)$ & $27.5(19.7)$ & $23.2(24.7)$ \\
\hline Peso para la edad, \% & $25.8(24.2)$ & $38.5(24.2)$ & $39.9(26.2)$ \\
\hline IMC para la edad, \% & $47.1(21.9)$ & $53.2(22.5)$ & $60.7(22.9)$ \\
\hline
\end{tabular}

* Valores son promedio (DE) 
Tabla 2. Consumo alimentario de escolares tarahumaras.

\begin{tabular}{|c|c|c|c|c|}
\hline & Tradicionales & Semi-tradicionales & Urbanos & Requerimientos \\
\hline Energía (kcal) & 1750 & 2485 & 2270 & $2015 *$ \\
\hline \% de Proteínas & 10 & 12 & 11 & $12 *$ \\
\hline$\%$ de Hidratos de carbono & 74 & 60 & 55 & 58 \\
\hline \% de Lípidos & 16 & 28 & 34 & 30 \\
\hline Fibras $(\mathrm{g})$ & 27 & 19 & 6 & \\
\hline Acido fólico $(\mu \mathrm{g})$ & 73 & 510 & 72 & $250 * *$ \\
\hline Vitamina $\mathrm{A}(\mu \mathrm{g})$ & 180 & 908 & 250 & $300-400 * *$ \\
\hline Calcio (mg) & 698 & 1281 & 249 & $400-800 * *$ \\
\hline Zinc (mg) & 2.8 & 7.2 & 1.6 & $1.8 * *$ \\
\hline Hierro (mg) & 18 & 74 & 7 & $13 * *$ \\
\hline
\end{tabular}

* FAO/OMS (1985)

** Bourges, H., Casanueva, E., Rosado, J. Recomendaciones de ingestión de Nutrimentos para la Población Mexicana (2005).

población exhibe una actividad física moderada. Estos niños tienen un retardo en su crecimiento y desarrollo debido a una alimentación insuficiente debido a la carencia de educación, servicios de salud y problemas en la cobertura de programas gubernamentales de ayuda alimentaria.

En los niños semi-tradicionales hay una fuerte ayuda alimentaria, la cual provoca una excesiva ingesta de micro-nutrimentos, contrastando esto con las deficiencias observadas en los niños tradicionales. Esto es un problema de focalización de apoyos, en el cual un grupo carece de elementos que resultan sobrantes para el otro.

Los escolares migrantes se encuentran mal nutridos, una tercera parte tiene anemia y la mitad de éstos tienen sobrepeso también. Este grupo mantiene el patrón cuatro de Popkin de transición alimentaria.

Cualquier proyecto de transición nutricional debe ser estudiado a profundidad y posteriormente generar las acciones a través de una estrategia de intervención que promueva la reducción en grasa corporal, incremente el consumo de frutas, verduras y fibra, así como bajar el sedentarismo por otras actividades.

\section{AGRADECIMIENTOS}

Estamos ampliamente agradecidos por el soporte de autoridades tradicionales tarahumaras, comunidad religiosa, civil y escolar, los traduc- tores raramuri-castellano, así como de los estudiantes que se formaron en este proyecto.

Principalmente se extiende nuestra gratitud para los 193 niños y padres voluntarios, que amablemente participaron en las pruebas.

Proyecto financiado por:

Universidad Iberoamericana, Ciudad de México; el Centro Nacional de Investigación Científica (CNRS). Francia, y el Consejo Nacional de Ciencia y Tecnología (CONACYT). México.

\section{REFERENCIAS}

1. RIVERA JA, MONTERRUBIO EA, GONZALEZCOSIO T, ET AL. Estado Nutricio de niños indígenas menores de 5 años en México: Resultados de una encuesta nacional probabilística. Salud Pública Mex 2003. 45,4: 1-11.

2. MONÁRREZ J, MARTÍNEZ H. Prevalence of malnutrition in children under 5 years in the municipality of Guachochi, Chihuahua. Salud Publica Mex 2000; 42,8:16.

3. OCHOA T, PÉREZ V, VELADOR A, LLAGUNO J. Prevalence of Malnutrition in children under five years in seven indian communities in the sierra tarahumara, Chihuahua. Ann Nutr Metab 2001; 45 (suppl 1): 582.

4. MONÁRREZ J, MARTÍNEZ H, MARTINEZ V, GREINER T. Nutritional status of indigenous children at boarding scools in northern México. Eur J Clin Nutr 2004; 532-40.

5. MONÁRREZ J, GREINER TA. Anthropometry in tarahumara indian women of reproductive age in northern Mexico: is overweight becoming a problem? Ecology of Food and Nutrition 2000; 39,6: 437. 
6. MCMURRY MP, ET. AL. Changes in lipid and lipoprotein levels and body weight in Tarahumara Indians after consumption of an affluent diet. N Engl J Med 1991; 12;325,1704-8.

7. PASQUET P, BALCAZAR M, RODRIGUEZ MF, HINOJOSA H, DE SANTIAGO S. Relationship between physical activity, aerobic capacity and body composition in school children from the Sierra Tarahumara, Mexico. In Proceedings of the18th International Congress of Nutrition,Nutrition Safari for Innovative Solutions, Vorster HH, Blaauw R, Dhansay MA, Kuzwayo PMN, Moeng L,Wentzel-Viljoen E (eds.), Edition electronique 2005. Karger, Bâle.

8. CABALLERO B, POPKIN B. The Nutrition Transition: diet and disease in the developing World. Academic Press 2002, p. 124-126.

9. MANUAL DEL ENTREVISTADOR: Encuestas de recordatorio de 24 horas y escala de Guttman. Departamento de Vigilancia Epidemiológica. Instituto Nacional de Ciencias Médicas y Nutrición "Salvador Zubirán"; Febrero 2006. México.

10. LOHMAN T, ROCHE A, MARTORELL R. Anthropometric standardization reference manual. Human Kinetics 1988; Champlaign, IL

11. HABICHT JP. Standardization of anthropometric methods in the field. PAHO Bull 1974; 76:375-384.

12. WORLD HEALTH ORGANIZATION. Physical Status: The use and interpretation of Anthropometry.
WHO Technical Report Service 845. WHO; 1995. Geneva.

13. WORLD HEALTH ORGANIZATION. Measuring Change in Nutritional Status: Guidelines for assessing the Nutritional Impact of supplementary feeding programs. WHO; 1983 Geneva.

14. Centres for Disease Control and Prevention/National Centre for Health Statistics. CDC Growth Charts: United States. Department of Health and Human Services; 2000. Hyattsville.

15. WHO/UNICEF/ONU, ED. Iron deficiency anemia, assessment, prevention and control: a guide for program managers. WHO/NHD/0.3. WHO; 2001. Geneva.

16. COHEN J, HASS J. Hemoglobin correction factors for estimating the prevalence of iron deficiency anemia in pregnant women residing at high altitudes in Bolivia. Rev Panam Salud Publica 1999; 6(6); .p.392-399.

17. PUYAU M, ADOLPH A, VOHRA F, BUTTE N. Validation and calibration of physical activity monitors in children. Obes Rev 2002; 10:150 - 157.

18. SHAMA-LEVY T, VILLALPANDO-HERNÁNDEZ S, RIVERA-DOMMARCO JA. Resultados de Nutrición de la ENSANUT 2006. Instituto Nacional de Salud Pública 2007; Cuernavaca, México.

Recepción: 13 de octubre de 2008 Aprobación: 17 de agosto de 2009

Usted puede comentar éste y otros artículos publicados en la Revista Chilena de Salud Pública, enviando un correo electrónico a revistasp@med.uchile.cl 